\title{
Several Inequalities on Moment of Uncertain Variables
}

\author{
Wei Li, Xiaosheng Wang* \\ School of Mathematics \& Physics, Hebei University of Engineering, Handan, China \\ Email address: \\ liwei066000@126.com (Wei Li), xswang@hebeu.edu.cn (Xiaosheng Wang) \\ ${ }^{*}$ Corresponding author
}

\section{To cite this article:}

Wei Li, Xiaosheng Wang. Several Inequalities on Moment of Uncertain Variables. Mathematics and Computer Science. Vol. 4, No. 2, 2019, pp. 57-62. doi: 10.11648/j.mcs.20190402.12

Received: July 26, 2019; Accepted: August 28, 2019; Published: September 21, 2019

\begin{abstract}
An uncertain variable is a Borel measurable function whose domain is uncertainty space and range is the set of real numbers. However, for many reasons, like the difficult of collecting data, the value of an uncertain variable is usually not easy to measure accurately. Hence many scholars study the estimation range of the value of an uncertain variable, and usually to estimate the upper or lower bounds of the moment of an uncertain variable is the primary idea. Many inequalities are established to estimate the above bounds, but there are still some problems on the estimation of the moment of uncertain variables. For instance, the even-order moment of an uncertain variable cannot be uniquely calculated at present. So the aim of this paper is to estimate the upper or power bounds of the moment of uncertain variables or the uncertain measure of an event by establishing several new inequalities. Firstly, we extend the Lyapunov inequality on uncertain variable and this inequality gives the upper bound of the even-order moment of an uncertain variable, and as a corollary, the lower bound of the above even-order moment is given. Then the inequality of arithmetic-geometry is proved, which estimates the lower bound of the expected value of an uncertain variable. After that, two equivalent inequalities are given, which can be used to judge the existence of the expected value of a function of an uncertain variable. Finally, as for two independent and identically distributed uncertain variables, the weakly symmetric inequalities are investigated to estimate the upper and lower bounds of the uncertainty distributions of the difference of these uncertain variables which implies the uncertain measures of several events. The above inequalities extend the application range of uncertain variable.
\end{abstract}

Keywords: Inequality, Uncertainty Theory, Upper or Lower Bound

\section{Introduction}

As we all know, it is usually difficult to precisely measure the value of a variable since many reasons, like the statistical errors. Hence many scholars study the estimation range of the value of a variable for some practical demands. Obviously the upper or lower bounds are firstly investigated to estimate the value of a variable. So many scholars have established many inequalities to estimate the upper or lower bounds of the value of the variables.

Especially in probability theory, some well-known inequalities have already been established, such as Jensen inequality, Markov inequality, Chebyshev inequality and so on. These inequalities provide the basic estimation of the upper or lower bounds of the moments of random variables or the probabilities of random events. For improving the traditional inequalities in probability theory, many scholars established different probability inequalities. For instance, Lin [1] established four equivalent inequalities and the inequality of arithmetic geometry on random variables in 2006. Then for pairwise independent random variables, Chen et al. [2] generalized a type of moment inequality in 2014. Later in 2015 , Gavrea [3] presented a Hadamard-type inequality to estimate the moments of continuous random variables. For the sums of negatively dependent random variables, Zhang [4] established Kolmogorov's and Rosenthal's inequalities in 2016. In 2017, Gibilisco et al. [5] presented an inequality to estimate the expected value of several positive random variables and proved the necessary and sufficient condition for this inequality held. And in 2017, Pelekis et al. [6] proved the Hoeffding's inequality and this inequality estimates the probability for the sums of dependent random variables as an upper bound. In 2018, Krebs [7] proposed a large deviation inequality for sums of random variables of the $\beta$-mixing form and proved the usefulness of applying the above inequality to 
the functional kernel regression model for dynamic forecasts. And Rio [8] improved the Doob's inequality and the so-called bounded differences inequality in 2018. In 2019, Maurer [9] gave a concentration inequality which extends Bernstein's inequality from sums to more general functions.

In 2007, in order to overcome the drawback of probability theory that relies on a large number of sample data, Liu [10] founded uncertainty theory which deals with the uncertainty based on expert's experimental knowledge. As one of the crucial parts of uncertainty theory, an uncertain variable is a Borel measurable function essentially. The domain of this function is the uncertainty space and the range is the set of real numbers. Similar to random variable, an uncertain variable is applied to describe the indeterminate phenomenon. Usually two factors, the expected value or the distribution, are chose to deal with an uncertain variable. Unfortunately, since the difficulties of collecting the experts' experimental data and the errors among different experts' experimental data, the value of an uncertain variable is usually difficult to measure accurately. Hence similar to the probability theory, scholars propose some inequalities to estimate the upper or lower bounds of the moments of an uncertain variable or the uncertain measure of an event.

Some classical inequalities on uncertain variables have already been given by Liu [10], such as Jensen's inequality, Markov inequality, Hölder's inequality and so on. Then based on these classical inequalities, some scholars proposed several new inequalities to estimate the uncertain variables. For instance, a moments and tails inequality on uncertain variables was proved by Yang [11] in 2011. Then Tian [12] extended Jensen's inequality, Liapounov theorem and Markov inequality for uncertain variables in 2011. Later in 2015, Zhu [13] discussed the conditions that the uncertainty model can be transformed into deterministic environment in the variational inequality problems. For the estimation of the value of an uncertain variable, many inequalities are established but there are still some problems on them. Hence in order to improve the methods to estimate the upper or lower bounds of moment of an uncertain variable or the uncertain measure of an event, this paper will continue to investigate several new inequalities on uncertain variable.

Firstly, in order to estimate an upper bounds of the even-order moment of an uncertain variable, a Lyapunov inequality is established and a corollary is mentioned for the lower bounds of the above even-order moment. Then when its uncertainty distribution is unknown, the expected value of an uncertain variable is usually difficult to obtain. So for estimating a lower bound of the expected value of an uncertain variable with unknown uncertainty distribution, the inequality of arithmetic-geometry is built and the condition for the equation is discussed. Next, a function of uncertain variables is usually an uncertain variable. When an uncertain variable's expected value is finite, whether the moment of a function of it exists is required to judge. For an exponential form of an uncertain variable, the existence of its expected value is discussed by two equivalent inequalities. Finally, for two independent and identical distributed uncertain variables, we know that the difference of these uncertain variables is still an uncertain variable. This difference's uncertainty distribution indicates the uncertain measure of the event. Hence two weakly symmetric inequalities are given to estimate the upper and lower bounds of the above uncertain measure.

The following is the structure of this paper. In Section 2, various inequalities are proposed and proved as a main result. Besides, a discussion is given in Section 3. And a conclusion is organized in Section 4.

\section{Results}

In this section, 4 groups of inequalities are given and proved.

\subsection{Lyapunov Inequality}

Let $\xi$ be an uncertain variable and let $M$ be the uncertain measure, the expected value of $\xi$ is defined by follow equation

$$
E[\xi]=\int_{0}^{+\infty} M\{\xi \geq x\} d x-\int_{-\infty}^{0} M\{\xi \leq x\} d x
$$

where one of the above two integrals on the right side of the equation is finite at least [10]. Then $E\left[\xi^{k}\right]$ represents the $k$ -th moment of $\xi$ where $k$ is a positive integer [10]. According to the value of $k$, there are two cases of calculating $E\left[\xi^{k}\right]$. When $k$ is an odd number, Liu [14] gave one method to calculate the $k$-th moment of $\xi$ via

$$
E\left[\xi^{k}\right]=\int_{0}^{+\infty}(1-\Phi(\sqrt[k]{x})) d x-\int_{-\infty}^{0} \Phi(\sqrt[k]{x}) d x
$$

where $\Phi$ is the uncertainty distribution of $\xi$. When $k$ is an even number, however, there has not been a theoretical method to uniquely calculate the $k$-th moment of $\xi$ yet. Hence we firstly give an upper bound of the $k$-th moment of $\xi$ by the following inequality.

Theorem 1 (Lyapunov Inequality). Let $\xi$ be a positive uncertain variable with $E\left[\xi^{p}\right]<+\infty$, for any real number $p>0$. Then for any real numbers $0<k \leq s$, we have

$$
\left(E\left[\xi^{k}\right]\right)^{\frac{1}{k}} \leq\left(E\left[\xi^{s}\right]\right)^{\frac{1}{s}}
$$

Proof. For any real numbers $0<k \leq s$, let

$$
g(x)=|x|^{\frac{s}{k}}
$$

Then, we have

$$
\begin{aligned}
& g^{\prime \prime}(x)=\frac{s(s-k)}{k^{2}}|x|^{\frac{s-2 k}{k}}>0, \\
& x \in(-\infty, 0) \cup(0,+\infty)
\end{aligned}
$$


Thus $g(x)$ is a convex function. For any positive uncertain variable $\xi$, replacing $\xi$ with $\xi^{k}$ in Jensen's inequality [15], we have

$$
\left(E\left[\xi^{k}\right]\right)^{\frac{s}{k}} \leq E\left[\left(\xi^{k}\right)^{\frac{s}{k}}\right]=E\left[\xi^{s}\right]
$$

Then, we obtain

$$
\left(E\left[\xi^{k}\right]\right)^{\frac{1}{k}} \leq E\left[\xi^{s}\right]^{\frac{1}{s}}
$$

Especially, letting $k=1$, then we have

$$
E[\xi] \leq\left(E\left[\xi^{s}\right]\right)^{\frac{1}{s}}
$$

Hence the theorem is proved.

The following result can be derived by this inequality:

$$
E\left[\xi^{k}\right] \leq\left(E\left[\xi^{s}\right]\right)^{\frac{k}{s}}
$$

That is, when $k$ is an even number and $s$ is an odd number, the upper bound of $E\left[\xi^{k}\right]$ can be estimated. Then a lower bound of the $k$-th moment of $\xi$ is proved by Corollary 1 .

Corollary 1. Let $\xi$ be a positive uncertain variable, and one assumes that if for any real numbers $k \geq 1, E\left[\xi^{k}\right]<\infty$, then we obtain

$$
E\left[\xi^{k}\right] \geq\left(E\left[\xi^{k-1}\right]\right)^{\frac{k}{k-1}}
$$

Proof. By the above Lyapunov inequality, we obviously obtain

$$
\left(E\left[\xi^{k}\right]\right)^{\frac{1}{k}} \geq\left(E\left[\xi^{k-1}\right]\right)^{\frac{1}{k-1}}
$$

where $k \geq 1$. Thus, we obtain

$$
E\left[\xi^{k}\right] \geq\left(E\left[\xi^{k-1}\right]\right)^{\frac{k}{k-1}}
$$

The result is proved.

\subsection{The Inequality of Arithmetic-Geometry}

By (1), it is obviously that the expected value of an uncertain variable depends on its uncertainty distribution. However, when the uncertainty distribution of an uncertain variable is unknown, the expected value, including high order moment, of it cannot be calculated. But when the logarithmic function of this uncertain variable has a known uncertainty distribution [15], the above expected value can be estimated by the following lower bound.

Theorem 2. (The Inequality of Arithmetic-Geometry) Let $\xi$ be a positive uncertain variable, then

$$
E[\xi] \geq e^{E[\ln \xi]}
$$

The above equation holds if $\xi$ is degenerate or $E[\ln \xi]=\infty$.

Proof. Case I: we assume that $E[\xi]<\infty$. Let $f(x)=x$, $h(x)=\ln x$, then for any $x>0$, we have

$$
f(x)>h(x)
$$

Hence for any real number $x>0$, we obtain

$$
\{\gamma \mid \xi(\gamma) \geq x\} \supset\{\gamma \mid \ln \xi(\gamma) \geq x\}
$$

Since the uncertain measure satisfies the monotonicity [10], we can obtain

$$
M\{\gamma \mid \xi(\gamma) \geq x\} \geq M\{\gamma \mid \ln \xi(\gamma) \geq x\}
$$

Then

$$
\int_{0}^{+\infty} M\{\xi \geq x\} d x \geq \int_{0}^{+\infty} M\{\ln \xi \geq x\} d x
$$

i.e.

$$
E[\ln \xi] \leq E[\xi]<\infty
$$

By Jensen's inequality [15], we have

$$
-\ln E[\xi] \leq E[-\ln \xi]
$$

which is equivalent to

$$
\ln E[\xi] \geq E[\ln \xi]
$$

Thus, we obtain

$$
E[\xi] \geq e^{E[\ln \xi]}
$$

Case II: when $\xi$ is degenerate that means it is not a variable but a crisp number [10], hence we have

$$
E[\xi]=e^{E[\ln \xi]}
$$

Case III: when $E[\ln \xi]=\infty$, we have $E[\xi]=\infty$. Then obviously

$$
E[\xi]=e^{E[\ln \xi]}
$$

In summary, the inequality is proved.

\subsection{Several Equivalent Inequalities}

Usually if a function is real-valued and measurable, then this function of uncertain variables is still an uncertain variable [10]. In general, before calculating the expected value of the above uncertain variable, we should judge that 
whether this expected value exists or not. The following inequalities give another criterion to judge the existence of the above expected value.

Theorem 3. Let $\xi$ be an uncertain variable and let $E[\xi]$ be the expected value of $\xi$. If $E[\xi]<\infty$, then the following description is equivalent.

(i) There exist a constant $H>0$, for any $|t|<H$, we have

$$
E\left[e^{t \xi}\right]<\infty
$$

(ii) There exist a constant $a>0$, such that

$$
E\left[e^{a|\xi|}\right]<\infty .
$$

Proof. If (ii) holds, let $H=a$. Hence for any $0 \leq|t|<H$, we get that

$$
\left\{\gamma \mid e^{t \xi(\gamma)} \geq x\right\} \subset\left\{\gamma \mid e^{a|\xi(\gamma)|} \geq x\right\}
$$

Since the uncertain measure satisfies the monotonicity, so we obtain

$$
M\left\{e^{t \xi} \geq x\right\} \leq M\left\{e^{a|\xi|} \geq x\right\}
$$

For any real number $t$, the function $M\left\{e^{t \xi} \geq x\right\}$ is monotone decreasing respect to $x \geq 0$. Hence the function $M\left\{e^{t \xi} \geq x\right\}$ is integrable on $x \in[0,+\infty)$, and

$$
\int_{0}^{+\infty} M\left\{e^{t \xi} \geq x\right\} d x \leq \int_{0}^{+\infty} M\left\{e^{a|\xi|} \geq x\right\} d x
$$

which implies

$$
E\left[e^{t \xi}\right] \leq E\left[e^{a|\xi|}\right]<\infty
$$

If (i) holds, we have

$$
E\left[e^{-|t| \xi}\right]<\infty
$$

and

$$
E\left[e^{|t| \xi}\right]<\infty
$$

i.e.

$$
E\left[e^{|t||\xi|}\right]<\infty
$$

Hence there exists a constant $\xi_{1}, \xi_{2}, \cdots, \xi_{n}$ that satisfies

$$
E\left[e^{a|\xi|}\right]<\infty .
$$

Then the theorem is proved.

\subsection{Weakly Symmetric Inequalities}

For the independent uncertain variables $\xi_{1}, \xi_{2}, \cdots, \xi_{n}$
[10], we have

$$
M\left\{\mathrm{I}_{i=1}^{n}\left(\xi_{i} \in B_{i}\right)\right\}=\bigwedge_{i=1}^{n} M\left\{\xi_{i} \in B_{i}\right\}
$$

where $\left|\xi^{s}\right|$ are any Borel sets of real numbers. In addition, if $\xi_{1}, \xi_{2}, \cdots, \xi_{n}$ have the same uncertainty distribution, they are said to be identically distributed.

For two independent uncertain variables which are identically distributed (iid), the difference of these uncertain variables is still an uncertain variable. Then the uncertainty distribution of the above difference can be interpreted as an event. The following inequalities offer the upper and lower bounds of this event.

Theorem 4. (Weakly Symmetric Inequalities) Let $\xi$ and $\xi^{\prime}$ be iid uncertain variable. Let $\xi^{s}=\xi-\xi^{\prime}$ be the difference of $\xi$ and $\xi^{\prime}$. Let real number $m_{\xi}$ be the median of $\xi$ and $m_{\xi}$ satisfies

$$
M\left\{\xi \geq m_{\xi}\right\} \geq \frac{1}{2}
$$

and

$$
M\left\{\xi \leq m_{\xi}\right\} \geq \frac{1}{2}
$$

Then for any real numbers $x$ and $c$, we have

$$
M\left\{\xi^{s} \geq x\right\} \geq M\left\{\xi-m_{\xi} \geq x\right\} \wedge \frac{1}{2}
$$

and

$$
M\left\{\left|\xi^{s}\right| \geq x\right\} \leq 2 M\left\{|\xi-c| \geq \frac{x}{2}\right\}
$$

Proof. Firstly, since $\xi$ and $\xi^{\prime}$ are iid, then we have

$$
m_{\xi}=m_{\xi}
$$

By [10], we obtain that $\xi^{s}, \xi-m_{\xi}, \xi^{\prime}-m_{\xi^{\prime}}$ and $\left(\xi-m_{\xi}\right)-\left(\xi^{\prime}-m_{\xi^{\prime}}\right)$ are uncertain variables. Hence

$$
\begin{aligned}
M\left\{\xi^{s} \geq x\right\} & =M\left\{\left(\xi-m_{\xi}\right)-\left(\xi^{\prime}-m_{\xi^{\prime}}\right) \geq x\right\} \\
\geq & M\left\{\xi-m_{\xi} \geq x, \xi^{\prime}-m_{\xi} \leq 0\right\} \\
& =M\left\{\xi-m_{\xi} \geq x\right\} \wedge M\left\{\xi^{\prime}-m_{\xi} \leq 0\right\} \\
\geq & M\left\{\xi-m_{\xi} \geq x\right\} \wedge \frac{1}{2} .
\end{aligned}
$$

Similarly, we know that $\left|\xi^{s}\right|, \xi-c, \xi^{\prime}-c$, and $\left|(\xi-c)-\left(\xi^{\prime}-c\right)\right|$ are uncertain variables where $c$ is any real number. Then 


$$
\begin{aligned}
M\left\{\left|\xi^{s}\right| \geq x\right\}=M\left\{\left|(\xi-c)-\left(\xi^{\prime}-c\right)\right| \geq x\right\} \\
\leq M\left\{\left\{|\xi-c| \geq \frac{x}{2}\right\} \cup\left\{\left|\xi^{\prime}-c\right| \geq \frac{x}{2}\right\}\right\} \\
\leq M\left\{|\xi-c| \geq \frac{x}{2}\right\}+M\left\{\left|\xi^{\prime}-c\right| \geq \frac{x}{2}\right\} \\
\quad=2 M\left\{|\xi-c| \geq \frac{x}{2}\right\} .
\end{aligned}
$$

Hence the inequalities are proved.

\section{Discussion}

The moment of uncertain variable is an important concept in uncertainty theory; many scholars have studied the methods to estimate the moment. Sheng and Kar [16] have given some formulas to calculate the moments of uncertain variables. In their work, when the uncertainty distribution of an uncertain variable is regular, then the $k$-th moment of this uncertain variable can be calculated by its inverse uncertainty distribution where $k$ is a positive integer. By Sheng and Kar's work, arbitrarily positive integer order moments of an uncertain variable could be accurately obtained on condition that its uncertainty distribution is regular.

However, by the definition of the regular uncertainty distribution, the monotonicity is required in order to obtain the inverse uncertainty distribution. Hence the Sheng and Kar's work is restricted by the above drawback.

Similar to Sheng and Kar's work, this paper is intent to extend the methods to estimate the moments of uncertain variables. Unlike their method, this paper established several inequalities to estimate the upper or lower bounds of the moments through the definition of moment, which avoided the requirement of the monotonicity of uncertainty distribution. The following are the significances of each inequality established in this paper.

For Lyapunov inequality and its corollary, when the odd-order moment of an uncertain variable is known, the even-order moment of this uncertain variable can be estimated by an upper bound and a lower bound. The above result firstly provides the method to estimate the even-order moments of an uncertain variable when its uncertainty distribution is not regular.

For the inequality of arithmetic-geometry, when the uncertainty distribution of an uncertain variable is unknown, a lower bound of its expected value can be given by the expected value of the logarithmic function of this uncertain variable. In the above result, the logarithmic function of an uncertain variable is still an uncertain variable, and its uncertainty distribution is assumed to be known.

For the two equivalent inequalities, it can be used to judge the existence of the expected value of an uncertain variable before calculating the above expected. Sometimes, when the existence is known, some conclusion can be obtained such as the runtime of an algorithm.

For the weakly symmetric inequalities, the uncertainty distribution of the difference of two iid uncertain variables can be estimated by the lower and upper bounds. When the uncertainty distribution is determined, the moment of the above difference could be estimated through the definition.

\section{Conclusion}

The primary innovation point of this paper is establishing several new inequalities on uncertain variable to estimate the upper or lower bounds of the moments of uncertain variable or the uncertain measure of events. Firstly, in order to estimate an upper bounds of the even-order moment of an uncertain variable, a Lyapunov inequality is established and a corollary is mentioned for the lower bounds of the above even-order moment. Then for estimating a lower bound of the expected value of an uncertain variable with unknown uncertainty distribution, the inequality of arithmetic-geometry is built and the condition for the equation is discussed. In addition, two equivalent inequalities on the expected value of an exponential uncertain variable are proposed to judge the existence of the above expected value. Finally, in order to estimate the upper and lower bounds of the uncertainty distribution of two independent and identically distributed uncertain variables, two weakly symmetric inequalities are given and proved. These inequalities improve the methods to estimate the value of an uncertain variable and extend the application range of uncertain variable.

\section{Acknowledgements}

Thanks to the support of National Natural Science Foundation of China (No.61873084) and the Foundation of Hebei Education Department (No. ZD2017016).

\section{References}

[1] Z. Lin, Z. Bai, Probability Inequality (in Chinese), Science Press, Beijing, 2006.

[2] P. Chen, B. Peng, S. H. Sung, The von bahrcesseen moment inequality for pairwise independent random variables and applications, Journal of Mathematical Analysis \& Applications 419 (2014) 1290-1302.

[3] B. Gavrea, A hermite-hadamard type inequality with applications to the estimation of moments of continuous random variables, Applied Mathematics and Computation 254 (2015) 92-98.

[4] L. Zhang, Rosenthal's inequalities for independent and negatively dependent random variables under sub-linear expectations with applications, Science China Mathematics 59 (2016) 751-768.

[5] P. Gibilisco, F. Hansen, An inequality for expectation of means of positive random variables, Annals of Functional Analysis 8 (2016) 142-151.

[6] C. Pelekis, J. Ramon, Hoeffdings inequality for sums of dependent random variables, Mediterranean Journal of Mathematics 14 (2017) 243. 
[7] J. Krebs, A large deviation inequality for $\beta$-mixing time series and its applications to the functional kernel regression model, Statistics and Probability Letters 133 (2018) 50-58.

[8] E. Rio, About Doob's inequality, entropy and Tchebichef, Electronic Communications in Probability 23 (2018) 1-12.

[9] A. Maurer, A Bernstein-type inequality for functions of bounded interaction, Bernoulli 25 (2019) 1451-1471.

[10] B. Liu, Uncertainty Theory, Springer Berlin Heidelberg, Berlin, Heidelberg, 2010, pp. 1-79. URL: https://doi.org/10.1007/978-3-642-13959-8_1.195 doi: 10.1007/978-3-642-13959-8_1.

[11] X. Yang, Moments and tails inequality within the framework of uncertainty theory, International Journal on Information 14 (2010) 2599-2604.
[12] J. Tian, Inequalities and mathematical properties of uncertain variables, Fuzzy Optimization \& Decision Making 10 (2011) $357-368$.

[13] Q. Chen, Y. Zhu, A class of uncertain variational inequality problems, Journal of Inequalities \& Applications (2015) 231.

[14] B. Liu, Uncertainty Theory, 4th edn, Springer-Verlag, Berlin, Heidelberg, 2015.

[15] B. Liu, Uncertainty Theory: A Branch of Mathematics for Modeling Human Uncertainty, Springer Publishing Company, Incorporated, Berlin, Heidelberg, 2010.

[16] Y. Sheng, S. Kar, Some results of moments of uncertain variable through inverse uncertainty distribution, Fuzzy Optimization \& Decision Making 14 (2015) 57-76. 